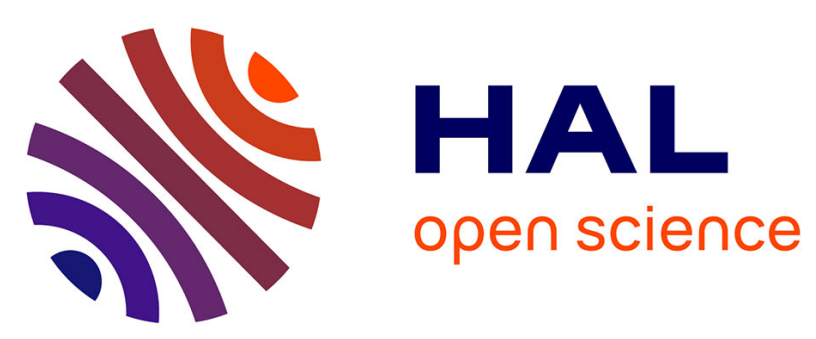

\title{
Lovelock, Gaïa et la pollution : un scientifique entrepreneur à l'origine d'une nouvelle science et d'une philosophie politique de la nature
}

Sébastien Dutreuil

\section{- To cite this version:}

Sébastien Dutreuil. Lovelock, Gaïa et la pollution : un scientifique entrepreneur à l'origine d'une nouvelle science et d'une philosophie politique de la nature. Zilsel : science, technique, société, 2017, 2, pp.19-61. 10.3917/zil.002.0019 . hal-01677761

\section{HAL Id: hal-01677761 https://hal.science/hal-01677761}

Submitted on 12 Jan 2018

HAL is a multi-disciplinary open access archive for the deposit and dissemination of scientific research documents, whether they are published or not. The documents may come from teaching and research institutions in France or abroad, or from public or private research centers.
L'archive ouverte pluridisciplinaire HAL, est destinée au dépôt et à la diffusion de documents scientifiques de niveau recherche, publiés ou non, émanant des établissements d'enseignement et de recherche français ou étrangers, des laboratoires publics ou privés. 


\title{
Lovelock, Gaïa et la pollution : un scientifique entrepreneur à l'origine d'une nouvelle science et d'une philosophie politique de la nature
}

\author{
Sébastien Dutreuil ${ }^{1}$
}

L'hypothèse Gaïa (HG) a été élaborée par James Lovelock dans les années 1960, et en collaboration avec Lynn Margulis dans les années 1970. En multipliant très tôt les genres de publication - revues scientifiques prestigieuses comme Nature, livres à destination du grand public et des scientifiques, presse généraliste, revues d'écologie politique - et en mêlant différents registres discursifs dans chacune de ces publications - Gaïa étant tantôt abordée comme un programme de recherche scientifique tantôt comme une philosophie de la nature -, Lovelock a contribué à singulièrement brouiller les repères sur le statut de Gaïa. Tandis que certains scientifiques l'ont considérée tantôt comme une hypothèse qu'il faudrait confronter directement aux faits empiriques, tantôt comme une théorie qu'il s'agirait d'élaborer à l'aide de modèles mathématiques et computationnels, d'autres ont abordé Gaïa comme un programme de recherche très large comprenant des revendications méthodologiques et ontologiques pour les sciences de la Terre et de l'environnement. Les philosophes et acteurs des mouvements environnementalistes l'ont lue comme une philosophie de la nature, visant à nous dire ce dont le monde est fait, à reconfigurer des concepts centraux comme ceux de vie, de nature et d'environnement, et à offrir une conception de la nature alternative à celle de la modernité.

La littérature sur HG s'est donc jusque-là concentrée soit sur des questions scientifiques et théoriques (c'est la littérature scientifique qui a émergé dans les années 1980 et 1990 dans le sillage du modèle Daisyworld), soir sur des questions philosophiques. Mais trop peu d'attention a été portée à l'histoire de l'élaboration d'HG et à la matrice scientifique, sociale et politique dense dans laquelle elle s'inscrit ${ }^{2}$. Cet article laisse de côté l'examen des pratiques et théories scientifiques et celui des déplacements philosophiques importants opérés par HG pour se concentrer sur des aspects importants de l'histoire d'HG.

Le récit historiographique dominant, que nous appellerons "exposé standard ", a été écrit par les détracteurs d'HG et a imposé deux idées. Premièrement, HG serait une hypothèse folklorique et tout à fait marginale n'ayant eu aucune influence réelle sur l'histoire des sciences et sur la manière dont nous nous représentons collectivement la Terre et la nature. Deuxièmement, HG, mettrait en avant une conception holiste, harmonieuse, ou animée de la nature et se voit ainsi connotée de manière positive sur le plan moral et politique.

L'examen de l'élaboration d'HG, de sa réception dans diverses disciplines scientifiques et auprès des mouvements environnementalistes et politiques, l'analyse de sa structure théorique et des prescriptions politiques de Lovelock m'ont conduit à soutenir l'exact opposé de ces deux thèses ${ }^{3}$. Premièrement, Gaïa n'a rien de marginal mais est au contraire tout à fait centrale en

\footnotetext{
1 Institut Max Planck d'Histoire des Sciences, Département II, seb.dutreuil@gmail.com. Ce travail est en partie issu d'une thèse dirigée par Jean Gayon et Philippe Huneman : je tiens à les remercier très sincèrement pour leur accompagnement exceptionnel de ce travail. Je voudrais également remercier Régis Briday, Lino Camprubi et Sebastien Grevsmühl pour la relecture de versions antérieures. Enfin, je remercie Hélène Guillemot et Nicolas Benvegnu en leur qualité d'examinatrice et d'examinateur de l'article pour leurs critiques et suggestions qui ont contribué à améliorer le texte originel.

2 Le livre de Michael Ruse se présente comme une histoire culturelle d'HG. Voir : Michael Ruse, The Gaia Hypothesis - Science on a Pagan Planet, Chicago, University of Chicago Press, 2013; Sébastien Dutreuil, "Review of : Michael Ruse, The Gaia hypothesis : science on a pagan planet », History and philosophy of the life sciences, vol. $36, \mathrm{n}^{\circ} 1$, p. 149-151.

3 Notamment dans ma thèse de doctorat consacrée à HG : Sébastien Dutreuil, Gaïa: hypothèse, programme de recherche pour le système terre, ou philosophie de la nature ?, Thèse, Université Paris 1
} 
sciences de la Terre et de l'environnement, mais aussi au sein des mouvements environnementalistes. C'est la raison pour laquelle elle doit être prise bien plus au sérieux qu'elle ne l'a été jusqu'à présent par les historiens, sociologues et philosophes des sciences et de l'environnement. Deuxièmement sa morale et sa politique n'ont rien de sympathique - pour des humanistes ou des tenants des principales sensibilités des mouvements environnementalistes -, ou, a minima, recèlent des problèmes qui doivent être repérés et explicités. Les propositions politiques récentes de Lovelock, exprimées dans La revanche de Gaïa, on suscité des réserves et critiques. Mais il faut en fait remonter en détail au contexte de l'élaboration d'HG dans les années 1960 pour comprendre les raisons historiques qui ont contribué à rendre Gaïa si centrale pour les sciences de l'environnement.

$C^{\prime}$ est ce retour qui nous permet de propose la thèse historique centrale de cet article: si on veut comprendre ce qu'est Gaïa et sa trajectoire historique, il faut d'abord et avant tout la voir comme l'élaboration d'un nouveau cadre général pour penser le phénomène de pollution globale, et non comme une réflexion spéculative et abstraite sur la nature de la vie ou la biologie évolutive.

Cette thèse remet en question aussi bien l'exposé standard que le second récit historiographique important sur HG, celui qui a été écrit et véhiculé par Lovelock lorsqu'il présente HG comme le fruit d'une réflexion spéculative sur la vie élaborée par un scientifique indépendant et solitaire.

La progression de l'article n'est pas chronologique, mais part des éléments les plus superficiels et connus pour arriver aux couches les plus profondes, recouvertes par des récits historiographiques qui se sont durablement installés. Après une présentation de l'exposé standard faisant d'HG une proposition pseudo-scientifique aux frontières de la biologie de l'évolution, nous remettons en question ce récit en montrant l'impact décisif d'HG en sciences de la Terre et de l'environnement et en écologie politique. C'est ce qui motive ensuite le retour à un examen détaillé du contexte d'élaboration d'HG.

\section{De l'élaboration de Gaïa a son influence scientifique}

\section{Élaboration et première réception de l'hypothèse Gaïa}

L'atmosphère a une composition chimique maintenue loin de l'équilibre thermodynamique par l'action des vivants ${ }^{4}$. C'est ce constat qui a conduit Lovelock à souligner l'influence considérable qu'ont les vivants sur leur environnement géologique ${ }^{5}$. De là, les auteurs d'HG ont proposé :

"l'hypothèse selon laquelle l'ensemble total des organismes vivants qui constituent la biosphère peut agir comme une seule entité pour réguler la composition chimique, le $\mathrm{pH}$ en surface et possiblement le climat. ${ }^{6}$

HG devait rendre compte d'un fait alors considéré comme surprenant: le maintien de I'habitabilité de la Terre pendant plus de 3 milliards d'années en dépit de perturbations externes.

Au début des années 1980, des critiques lapidaires ont été formulées par des biologistes de l'évolution Richard Dawkins et Ford Doolittle ${ }^{7}$ : certaines formulations d'HG semblent

Panthéon-Sorbonne, Paris, 2016.

4 James Lovelock, "A physical basis for life detection experiments ", Nature, vol. 207, n 4997, 1965, p. 568-570 ; Dian R. Hitchcock et James Lovelock, " Life detection by atmospheric analysis », Icarus, vol. 7, 1967, p. 149-159.

5 James Lovelock, "Gaia as seen through the atmosphere ", Atmospheric Environment, n 6, 1972, p. 579-580.

6 James Lovelock et Lynn Margulis « Atmospheric homeostasis by and for the biosphere : the Gaia hypothesis. ", Tellus, vol. 26, $n^{\circ} 1,1974$, p. 3. 
téléologiques, puisqu'elles laisseraient entendre que la "fonction " des vivants est de réguler l'environnement; et HG semble présupposer l'existence d'une forme d'altruisme planétaire, chacun des vivants contribuant, par son action, au bien commun - or la possibilité même d'un tel altruisme entrait en conflit avec les conceptions de l'époque en théorie de l'évolution.

On peut montrer à partir d'une analyse historique détaillée que ces deux critiques reposent sur des lectures superficielles et erronées $\mathrm{d}^{\prime} \mathrm{HG}^{8}$. Mais, dans le sillage de ces critiques, formulées par des scientifiques ayant une audience large, un exposé standard a installé de manière durable et profonde, auprès d'une partie importante de la communauté scientifique et du grand public, l'idée qu'HG ne serait qu'une hypothèse folklorique ou pseudo-scientifique n'ayant intéressé, au mieux, que les mouvements New Age.

Cet exposé standard affirme que s'il y a eu une controverse scientifique, elle a concerné au premier chef les biologistes de l'évolution. Or, on peut montrer à partir d'une analyse quantitative de la réception d'HG que, pour l'essentiel, les biologistes de l'évolution n'ont pas critiqué HG : ils s'en sont simplement désintéressés après les critiques lapidaires de Doolittle et Dawkins. En outre, un examen du contenu et des lieux de publication des premiers textes sur HG ne laisse aucun doute sur le fait que ses auteurs envisageaient une contribution au sein des sciences de la Terre (climatologie, géochimie et histoire de la Terre) et à la marge de la biologie (exobiologie), et non au sein de la biologie de l'évolution. Or, si HG n'a nullement intéressé les biologistes de l'évolution, elle a eu une influence particulièrement profonde, puissante et durable sur la trajectoire historique des sciences de la Terre et de l'environnement.

\section{L'apport central de Gaïa : la constitution d'un nouvel objet de savoir pour les sciences de la Terre et de l'environnement}

On comprend mal le succès et la réception très large d'HG si on l'aborde comme une "hypothèse scientifique ", comprise comme une proposition générale qu'il s'agirait de tester en la confrontant aux faits empiriques. Avant d'être le nom d'une hypothèse, "Gaïa " a d'abord été celui d'une entité, mise en avant par Lovelock dans un texte intitulé " Gaia as seen through the atmosphere $"$ :

"L'objectif de cette lettre est de suggérer que la vie à un stade précoce de son évolution a acquis la capacité de contrôler l'environnement global de manière à répondre à ses besoins et que cette capacité a persisté et est toujours activement utilisée. Dans cette perspective la somme totale des espèces est davantage qu'un simple catalogue, "La Biosphère", et comme d'autres associations en biologie est une entité avec des propriétés qui sont davantage que la simple somme de ses parties. Une créature si grande, même si elle est seulement hypothétique (...), a besoin d'un nom ; je dois à M. William Golding la suggestion d'utiliser la personnification grecque de la Terre mère, "Gaïa". „"

Deux modes principaux de définition de Gaïa comme entité ont été proposés. Le premier désigne l'ensemble des individus vivants: Gaïa, c'est "la vie ». C'est le sens de la citation reproduite ci-dessus. Plusieurs termes ont ensuite été utilisés de manière interchangeable : « la vie ${ }^{10}$ (singulier) ; "la biosphère ${ }^{11}$; « la somme totale des espèces ${ }^{12}$ »; " l'ensemble total

\footnotetext{
7 Ford W. Doolittle "Is nature really motherly ", CoEvolution Quarterly, n² 29, 1981, p. 58-63 ; Richard Dawkins, The extended phenotype: The gene as the unit of selection, Oxford, Oxford University Press, 1982.

8 Dutreuil, Gaïa. Op. cit.

9 Lovelock, « Gaia as seen ». Op. cit., p. 579.

10 Lovelock, "Gaia as seen ». Op. cit., p. 579 ; Lovelock et Margulis, "Atmospheric homeostasis ». Op. cit., p. 2.

11 Lovelock et Margulis, «Atmospheric homeostasis ». Op. cit., p. 2.

12 Lovelock, « Gaia as seen ». Op. cit., p. 579.
} 
des organismes qui constituent la biosphère $»^{13}$; « le biote $»^{14}$. Le second a été introduit dans le texte emblématique de 1974 :

"Désormais, le mot "Gaïa" sera utilisé pour décrire la biosphère et toutes les parties de la Terre avec lesquelles elle interagit activement pour former cette hypothétique nouvelle entité avec des propriétés qui ne peuvent être prédites de la somme de ses parties. $)^{15}$

Ici Gaïa est définie comme l'écosystème planétaire. Gaïa a ensuite été appelée "système Terre » par ceux qui ne voulaient pas être partie liées avec le label « Gaïa ».

C'est précisément la reconnaissance d'une nouvelle entité, Gaïa ou le système Terre, qui a présidé à la constitution des sciences du système Terre à partir des années 1980 . Le label "sciences du système Terre " désigne une reconfiguration importante et profonde des sciences de la Terre visant à regrouper des disciplines auparavant séparées - comme la géochimie, la climatologie, l'écologie globale, l'océanographie, etc. -, en les rassemblant autour de l'étude d'un même objet: le système Terre. Cette reconfiguration, initiée aux États-unis, a rapidement connu une envergure internationale. L'un de ses héritages les plus connus est la constitution de l'International Geosphere-Biosphere Programme (IGBP), une institution sous l'égide de I'ICSU (International Council of Scientific Unions) visant à fédérer les disciplines mentionnées ci-dessus autour de l'étude des changements globaux.

L'histoire d'HG illustre de manière magistrale comment des scientifiques pourtant sérieux et attentifs à $\mathrm{HG}$, comme Kirchner ou Tyrrell ${ }^{16}$, ont pu passer à côté de l'essentiel de l'intérêt d'HG en la forçant à rentrer dans le cadre étroit d'une "hypothèse ", suivant la conception poppérienne de l'activité scientifique. Contrairement à ce que son nom indique, HG ne doit pas être conçue comme une "hypothèse ": son apport historique décisif a été de constituer un nouvel objet de savoir autour duquel se sont constituées les sciences du système Terre.

C'est précisément pour cet apport que Lovelock a reçu en 2006 la plus haute distinction de la Société Géologique de Londres, la médaille Wollaston :

"Même dans l'histoire illustre de la médaille majeure de la Société, décernée pour la première fois à William Smith en 1831, il est rare d'être à même de dire que le récipiendaire a ouvert un champ entier et nouveau d'étude en science de la Terre. Mais c'est le cas du gagnant de cette année, James Lovelock. (...). La vue de la planète et de la vie qui y vit comme un système complexe unique (...) est ce qui a donné naissance au champ que nous connaissons maintenant comme 'Science du Système Terre' (...). II est difficile de trop souligner le caractère unifiant de cette vision du monde holiste, qui a brisé des barrières disciplinaires artificielles qui ont existé depuis la fin du $18^{\mathrm{e}}$ et le début du $19^{\mathrm{e}}$ siècle quand les Sociétés comme celle-ci ont été formées pour la première fois, et la formidable richesse des connaissances qui ont découlé de la multidisciplinarité qui a suivi. $»^{17}$

13 Lovelock et Margulis, «Atmospheric homeostasis ». Op. cit., p. 3.

14 Hitchcock et Lovelock, "Life ». Op. cit., p. 149 ; Lovelock, " Gaia as seen ». Op. cit., p. 579.

15 Lovelock et Margulis, «Atmospheric homeostasis ». Op. cit., p. 3.

16 James Kirchner " The Gaia hypothesis : can it be tested. ", Reviews of Geophysics, vol. 27, n 2, 1989, p. 223-235; Toby Tyrrell, On Gaia : A Critical Investigation of the Relationship Between Life and Earth, Princeton, Princeton University Press, 2013.

17 Geological Society of London, Wollaston medal, James lovelock, URL : https://www.geolsoc.org.uk/About/History/Awards-Citations-Replies-2001-Onwards/2006-Awards-Citati ons-Replies, 2006, consulté le 22 juin 2017. Bien sûr, la citation de la Geological Society ne constitue en rien une preuve historiographique sérieuse permettant de saisir les relations entre Gaïa et les sciences du système Terre. Il semble en principe possible d'objectiver l'influence d'une idée - comme HG - ou d'un acteur - comme Lovelock ou Margulis - sur un ou des champs scientifiques - comme la constitution des sciences du système Terre, l'évolution des problèmes et objets de la climatologie, de la géochimie, de l'écologie, et de l'histoire de la Terre -, en s'appuyant sur des outils de bibliométrie quantitative. Mobiliser de tels outils pour retracer quantitativement l'émergence et l'histoire des sciences du système Terre constitue ainsi un horizon important de notre travail. Mais précisément parce que le label « Gaïa » 
Les sciences du système Terre résultent d'une effervescence institutionnelle déployée à un haut niveau de la structuration de la recherche américaine et internationale ${ }^{18}$. Lovelock s'est toujours tenu à l'écart des comités et institutions organisant la recherche et n'a pas pris part personnellement à cette effervescence institutionnelle. Mais nombre des acteurs impliqués dans celle-ci et ayant ensuite eu un rôle important pour le cadrage programmatique et théorique des sciences du système Terre ont été très largement influencés par Lovelock et HG.

Contrairement à ce que le récit standard laisse entendre, HG est donc loin d'être une hypothèse folklorique aux frontières de la science: elle a eu une influence large sur la trajectoire des sciences de la Terre et de l'environnement.

\section{Une philosophie de la nature aux relations ambivalentes avec l'écologie politique et les mouvements environnementalistes}

Le récit standard qui a discrédité HG ne se trompe pas seulement lorsqu'il se prononce sur la communauté scientifique au sein de laquelle HG s'insère, mais également lorsqu'il envisage la réception d'HG auprès du grand public via les mouvements "New Age ». Or, I'histoire scientifique d'HG est inextricablement liée à celle de l'élaboration et de la diffusion d'HG auprès des mouvements environnementalistes et de l'écologie politique. Ainsi, si le célèbre climatologue Stephen Schneider ${ }^{19}$ et la spécialiste d'écologie microbienne Penelope Boston prennent l'initiative d'organiser une conférence internationale consacrée à Gaïa en 1988, sous l'égide de I'AGU (l'American Geophysical Union, structurant les sciences de la Terre internationale), c'est parce qu'ils voulaient « que le débat sur HG se tienne à l'endroit qui lui appartenait - à l'intérieur du monde de la science standard, pas simplement à la télé ou dans des magasines de la contre-culture comme Coevolution Quarterly. ${ }^{20}$

a eu mauvaise presse dans la littérature scientifique, un tel travail de bibliométrie quantitative sous-estime grandement l'ampleur du réseau d'HG. Une cartographie de la réception d'HG doit donc en passer par un travail d'analyse qualitative et quantitative plus fin que ce que permettent les recherches par mots clefs. Notre travail est basé sur une analyse des archives de Lovelock [Archives de James Lovelock, 1935-2011, 78 boîtes, Science Museum Library and Archives, Science Museum at Wroughton, Hackpen Lane, Wroughton, Angleterre] ; des entretiens avec les acteurs qui l'ont côtoyé et ont été inspirés par Lovelock et Gaïa ; le suivi de ses conférences et des institutions avec lesquelles il a collaboré, y compris de manière informelle; l'histoire de l'évolution des problèmes dans les champs scientifiques concernés par HG, etc. Nous renvoyons à Dutreuil, Gaïa. Op. cit., pour les détails de la démonstration et l'analyse des relations complexes entre Gaïa et les sciences du système Terre.

18 Écrire l'histoire des sciences du système Terre dépasse le cadre de cet article. Conway a abordé cette question depuis une histoire des sciences de l'atmosphère à la NASA : Erik M. Conway, Atmospheric science at NASA : a history, Baltimore, John Hopkins University Press, 2008 ; Guillemot et Dahan depuis des histoires de la modélisation en climatologie : Hélène Guillemot, La modélisation du climat en France des années 1970 aux années 2000 : histoire, pratiques, enjeux politiques, Thèse, EHESS, Paris, 2007 ; Amy Dahan, "Putting the Earth System in a numerical box? The evolution from climate modeling toward global change ", Studies in History and Philosophy of Science Part B : Studies in History and Philosophy of Modern Physics, vol. 41, n 3, 2010, p. 282-292; Kwa depuis une histoire des programmes d'écologie et de I'IGBP: Chunglin Kwa "Local ecologies and global science discourses and strategies of the International Geosphere-Biosphere Programme. "Social Studies of Science, vol. 35, $\mathrm{n}^{\circ}$ 6, 2005, $\mathrm{p}$. 923-950 ; Grinevald depuis une histoire de l'écologie globale et des sciences de la biosphère : Jacques Grinevald, "L'effet de serre de la biosphère ", Stratégies énergétiques, Biosphère et Société, 1990. Pour une analyse éclairante d'ouvrages importants sur l'histoire des sciences de l'environnement global, voir Lino Camprubi, "The invention of the global environment ", Historical Studies in the Natural Sciences, Vol. 42, n², 2016 p. 243-251.

19 Fondateur de la revue Climatic Change et acteur décisif pour l'export de la question climatique auprès du grand public.

20 Stephen H. Schneider, Science as a contact sport: inside the battle to save earth's climate, Washington, D.C., National Geographic Books, 2009, p. 66. 
Le récit standard fait souvent référence aux reprises "New Age " de Gaïa, sans que cette expression, utilisée comme épouvantail dans le débat scientifique, n'emporte avec elle de caractérisation sociologique précise. En renvoyant de manière lointaine et vague à un folklorisme néo-païen marginal, l'exposé standard d'HG empêche de voir l'influence qu'HG a eue depuis les années 1970 au sein des arènes les plus influentes des mouvements environnementalistes. II est un deuxième quiproquo, plus profond que celui sur le New Age, qu'il faut clarifier. Précisément parce que Gaïa a été conçue comme une philosophie bienveillante de la nature et est étroitement associée à divers courants importants de l' environnementalisme, beaucoup sont restés entièrement aveugles au fait pourtant massif suivant lequel Lovelock ne partage pas, et loin s'en faut, les idéaux et les positions politiques de ces mêmes mouvements.

\section{Néo-paganisme ou contreculture environnementale américaine et écologie politique?}

À force de dénoncer de manière abstraite les «mouvements New Age » auxquels Gaïa serait liée, les scientifiques et commentateurs ont considérablement brouillé les pistes historiques. $\mathrm{Si}$ bien que Michael Ruse peut mettre au centre de son histoire portant sur la réception de Gaïa dans le grand public, la figure d'Oberon Zell-Ravenheart, considéré comme l'un de ceux qui a popularisé le terme "néo-païen » à la fin des années 1960 et les a fédérés autour d'une revue (Green egg) :

"Cet homme remarquable se décrit lui-même sur son site web comme un "psychologue transpersonnel, métaphysicien, naturaliste, théologien, shaman, auteur, artiste, sculpteur, lecteur, enseignant" et également comme un "initié à l'Église Égyptienne de la Source Éternelle" ainsi qu'un "Prêtre de la confrérie d'Isis". " ${ }^{21}$

Or ce $n^{\prime}$ est pas au sein de ce groupe restreint et marginal ${ }^{22}$, quelque part entre sorcellerie et rites néo-païens, que l'hypothèse Gaïa de Lovelock et Margulis a circulé23. En revanche, Gaïa a diffusé auprès du grand public via des canaux de diffusion autrement plus amples et auprès de groupes très différents du néo-paganisme : la contre-culture environnementale californienne et américaine dont Stewart Brand et le Whole Earth Catalog sont les figures de proue dans les années 1960 et 1970 ; mais aussi l'écologie politique britannique, via Edward Goldsmith, fondateur de la revue The Ecologist (et sa version française L'écologiste) et Satish Kumar, fondateur de la revue Resurgence et du Schumacher College dans le Devon.

Le Whole Earth Catalog, un manuel destiné aux mouvements communautaires, s'est vendu au début des années 1970 a plusieurs millions d'exemplaires. La revue qui lui a succédé, Coevolution Quarterly, a publié en une plusieurs textes sur Gaïa, de Lovelock et Margulis et de théoriciens des systèmes, jusqu'à la recension critique de Doolittle ${ }^{24}$. Le réseau qui animait ces revues de la contreculture environnementale américaine a ensuite eu une influence décisive sur l'élaboration de la cyberculture contemporaine ${ }^{25}$. Edward Goldsmith et sa revue The Ecologist ont joué un rôle structurant pour l'écologie politique ${ }^{26}$. Le Schumacher College, où Lovelock a donné les conférences d'ouverture en 1991, a accueilli divers acteurs et théoriciens des mouvements environnementalites.

21 Ruse, The Gaia. Op. cit., p. 142.

22 Le néo-paganisme américain regroupait de l'ordre de 100000 personnes dans les années 1970. Cf. Margot Adler, Drawing down the moon: Witches, druids, goddess-worshippers, and other pagans in America, New York, Penguin Books, 2006.

23 Oberon joue la démarcation vis-à-vis de cette version de Gaïa, et Lovelock rappelle que si Oberon l'a contacté à l'époque, le scientifique refusait explicitement d'être associé au néo-paganisme.

24 Doolittle, «Is nature ». Op. cit.

25 Fred Turner, From counterculture to cyberculture : Stewart Brand, the Whole Earth Network, and the rise of digital utopianism, Chicago, University Of Chicago Press, 2010.

26 Sur Goldsmith et son écologie conservatrice et réactionnaire, voir : Jean Jacob, Histoire de l'écologie politique, Paris, Albin Michel, 1999. 
Brand, Kumar et Goldsmith sont de longue date des amis proches de Lovelock et leurs revues et leurs livres ont donné un très large écho à Gaïa. Avec Brand, Lovelock partageait un goût pour la cybernétique et les technologies de petite échelle et d'information. Au sein de l'écologie profonde de Goldsmith, c'est la conception holiste et unitaire de la Terre qui a suscité un intérêt.

Lovelock s'est également lié d'amitié avec des acteurs politiques impliqués dans la cause environnementale comme Jonathon Porritt (président du Ecology party à ses débuts puis de Friends of The Earth en Angleterre dans les années 1980) ou le diplomate anglais Crispin Tickell. Dans les années 1980, enfin, Lovelock a été conseiller ponctuel de Thatcher sur des questions climatiques.

Pour conclure, contrairement à ce qui est souvent affirmé, si HG a largement circulé auprès du grand public ce n'est pas via et au sein du néo-paganisme américain mais de la contreculture environnementale américaine et de l'écologie politique britannique.

Plus récemment, dans le sillage de l'effervescence engendrée par les discussions du concept d'anthropocène au sein des sciences humaines et sociales, Gaïa a suscité l'intérêt de philosophes aux orientations théoriques très variées, de l'éthique environnementale américaine de Callicott ${ }^{27}$ à l'anthropologie politique de la nature de Latour ${ }^{28}$ - après avoir suscité les réserves et critiques d'autres courants, comme l'écologie sociale ${ }^{29}$.

\section{Les prescriptions politiques de Lovelock : les CFCs, le DDT et les verts}

Compte tenu du rôle structurant de la figure de Gaïa au sein de divers courants de l'écologie politique, Lovelock est souvent présenté comme un penseur important de ces courants et comme le parrain des mouvements environnementalistes. Cette association très forte rend de nombreux lecteurs de Lovelock aveugles à ses prises de positions pourtant très claires.

Un livre de Lovelock a particulièrement fait réagir, aussi bien les climatologues que les ONG environnementalistes: La revanche de $\mathrm{Gaïa}^{30}$. Dans ce livre, Lovelock expose une situation environnementale catastrophique à une échelle globale et préconise des mesures d'urgence: solutions de géoingénierie, suspensions des droits humains et de la démocratie, reprise d'un néo-malthusianisme (si l'on ne trouve pas de moyens de réduire la population de manière "volontaire ", c'est "Gaïa ... qui opérera la réduction de la population et éliminera ceux qui enfreignent ses règles ${ }^{31}$ ). Récemment encore, Lovelock soutenait par exemple que l'exploitation des gaz de schiste constituait une excellente voie, dans laquelle la Grande-Bretagne devrait s'engager ${ }^{32}$. À partir du milieu des années 2000, Lovelock reprend une histoire inventée de toutes pièces dans les années 1990 par des marchands de doute ${ }^{33}$ : Rachel $^{2}$ Carson serait responsable de millions de morts de la malaria dans les pays tropicaux en ayant

\footnotetext{
27 John B. Callicott, Thinking like a planet : the land ethic and the earth ethic, Oxford, Oxford University Press, 2014.

28 Bruno Latour, Face à Gaïa : huit conférences sur le nouveau régime climatique, Paris, La Découverte, 2015.

29 Par exemple, David Pepper, Eco-socialism : from deep ecology to social justice, London, Routledge, 1993. La cartographie de la réception d'HG au sein des sciences humaines et sociales dépasse le cadre de cet article ; cf. Dutreuil, Gaïa. Op. cit., p. 223-225.

30 James Lovelock, The revenge of Gaia, London, Penguin Books, 2006.

31 Lovelock, 2006, Ibid., p. 181. Sur ce point, voir également Sebastian Grevsmühl, La Terre vue d'en haut : I'invention de l'environnement global. Paris, Seuil, 2014.

32 Leo Hickman " James lovelock on shale gas and the problem with "greens" ", The Guardian, 15 juin 2012.

${ }^{33}$ Sur l'élaboration de ce hoax, voir : Naomi Oreskes et Erik M. Conway, Merchants of doubt: how a handful of scientists obscured the truth on issues from tobacco smoke to global warming, New York, Bloomsbury Press, 2010, chapitre 7.
} 
contribué à interdire le DDT, ce qui aurait empêché de contrôler cette maladie, tandis que le caractère cancérigène du DDT ne serait qu'une rumeur ${ }^{34}$.

On ne comprend absolument rien à ces prises de position si on les interprète comme des tremblements inquiétants et tardifs d'un penseur qui se serait éloigné de la voie gaïenne qu'il aurait tracée des décennies auparavant.

Pour des raisons examinées dans les sections suivantes, Lovelock, par ses travaux scientifiques, s'est retrouvé au centre de nombreuses controverses environnementales, comme le changement climatique, ou l'ozone et l'interdiction des CFCs. Dans ses ouvrages sur Gaïa, Lovelock s'est ainsi très régulièrement exprimé sur ces controverses et a eu l'occasion de dire tout le mal qu'il pense des "verts ».

II a en effet toujours entretenu des relations ambivalentes avec ceux qu'il appelle "les verts ". Il est difficile de rattacher des noms ou des institutions derrière ce label que Lovelock utilise souvent ${ }^{35}$. Lovelock utilise le plus souvent « vert " pour renvoyer à un portrait abstrait. Pour lui, le vert idéal-typique est un personnage qui ne connaît rien à la chimie, voire à la science en général; qui, ne connaissant rien à la chimie, développe une crainte "idéologique ${ }^{36}$ et "fanatique ${ }^{37}$ de tout produit chimique ou du nucléaire ignorant la sagesse paracelsienne suivant laquelle le poison c'est la dose et ignorant l'existence d'équilibres gaïens; qui est plus inquiet de la santé des humains et des cancers hypothétiquement causés par des substances chimiques que des problèmes réels menaçant la Terre. Le vert est un urbain ne se souciant pas de détruire la campagne avec des champs d'éoliennes; et qui, s'inquiétant à tort des menaces potentielles sur les humains, et enrôlant avec lui le grand public, des idéologues et une machinerie bureaucratique et politique, freine la croissance et la liberté d'entreprendre, quand "le vert» ne mobilise pas des arguments scientifiquement discutables pour critiquer le capitalisme.

Si Gaïa a connu un large succès auprès des mouvements environnementalistes et si Lovelock était très proche de certains acteurs au centre de ces mouvements, les positions de Lovelock, elles, s'éloignent le plus souvent de celles de l'écologie politique.

\section{Les auteurs de Gaiia et le contexte de l'élaboration}

Pour éclairer la réception scientifique d'HG et sa diffusion dans les mouvements environnementalistes mais aussi les prises de position politiques de Lovelock, il est indispensable de revenir en détail sur le contexte de l'élaboration d' $\mathrm{HG}^{38}$.

34 Lovelock, The revenge. Op. cit., p. 138 ; James Lovelock, The Vanishing Face of Gaia, New York, Basic Books, 2009 p. 115, 124 ; James Lovelock, A Rough Ride to the Future, London, Penguin Books, 2014, p. 126.

${ }^{35}$ Cela est difficile non seulement parce que le mouvement environnementaliste est éminemment hétérogène, mais également parce que les attaches intellectuelles et institutionnelles ainsi que les liens d'amitié que Lovelock entretient de décennies en décennies avec certains acteurs incontournables de l'écologie politique contribuent à brouiller certains repères. Greenpeace est dans son viseur , cf. Lovelock, The Vanishing. Op. cit., p. 31 ; Lovelock, A rough. Op. cit., p. 84. Lovelock a eu sa carte à Friends of the Earth, vraisemblablement à la suite d'une invitation à adhérer par Porrit en 1986 [lettre de Porritt à Lovelock du 21 mars 1985, archives de Lovelock], mais les membres des Amis de la Terre s'étranglent lorsque Lovelock suggère dans une émission de télévision en 1988 que les Amis de la Terre devraient être appelées "Les amis des gens » compte tenu de leur orientation [lettre de Stewart Boyle (responsable de campagne à Friends of The Earth) à Lovelock du $1^{\mathrm{er}}$ août 1988, archives de Lovelock].

36 Lovelock, A rough. Op. cit., p. 126.

37 James Lovelock, Homage to Gaia : the life of an independent scientist, Oxford, Oxford University Press, 2000, p. 205.

38 Les éléments biographiques présentés sur Lovelock proviennent : de l'autobiographie de Lovelock: Lovelock, 2000, Ibid.; des nombreux entretiens et documentaires à son sujet [e.g. John Groom. Goddess of the Earth. NOVA Documentary, 1986 ; Anne Lacking. Beautiful minds, Series 1, James Lovelock. BBC, 2010 ; Paul Merchant, Lovelock, James. An oral history of British science, British Library, 2010. La seule biographie publiée est celle de Gribbin et Gribbin : John Gribbin et Mary Gribbin, James Lovelock : in 


\section{Lovelock : ingénieur et chimiste}

Après le lycée, Lovelock commence à travailler comme technicien dans un laboratoire de chimie: c'est là qu'il a acquis ce qu'il appelle son obsession pour la précision des mesures. Il est recruté au Medical Research Council de Londres en 1941, après son diplôme en chimie de l'université de Manchester. Il y effectue divers travaux mêlant chimie, virologie, bactériologie, expérimentation animale. Il contribue à l'élaboration des protocoles pour la mise au point de nouveaux instruments de mesure. Pendant près de vingt ans, il travaille ainsi dans divers domaines, chacun apportant son lot d'articles publiés dans Nature: la transmission $d^{\prime}$ infections ${ }^{39}$, l'effet de la chaleur sur les tissus et sur la coagulation sanguine ${ }^{40}$, des travaux pionniers en cryobiologie toujours cités aujourd'hui - effet de la congélation sur les tissus et les cellules $^{41}$, résurrection de hamsters congelés ${ }^{42}$, divers travaux en biochimie et biophysique ${ }^{43}$.

Avant l'élaboration d'HG, entre le début des années 1940 et le début des années 1960, Lovelock est donc ingénieur et chimiste dans le domaine biomédical. C'est précisément pour ses talents d'ingénieur et sa capacité à inventer des appareils de détection qu'il est recruté par la NASA en 1961 comme scientifique consultant, initialement pour la mise au point de chromatographes et d'autres instruments de mesure prévus pour les missions Surveyor et Mariner $B$ devant explorer la Lune et Mars. C'est dans ce cadre que Lovelock sera amené à réfléchir à un critère de détection de vie sur d'autres planètes. Au moment de l'élaboration de Gaïa, Lovelock n'est donc en rien un marginal éloigné de l'activité scientifique : il a derrière lui une carrière scientifique brillante.

Lovelock n'était pas un théoricien ou un penseur philosophique retiré du monde. Dans les années 1960 et 1970, Gaïa était pour lui un travail à temps partiel. Son temps est occupé à effectuer des mesures de chimie à la surface du globe : des mesures de chimie de l'atmosphère et des océans à bord de navires océanographiques traversant l'Atlantique, à pied dans sa campagne anglaise et irlandaise, ou à bord d'avion supersoniques du MetOffice britannique ${ }^{44}$.

L'ancrage de Lovelock dans le domaine de la chimie éclaire tout ce qui le sépare, dans ses prises de position, des figures associées à l'émergence des mouvements environnementalistes, souvent ancrées dans le domaine de l'écologie scientifique, comme Rachel Carson ou Barry

search of Gaia, Princeton, Princeton University Press, 2009. Cette biographie hagiographique livre peu d'éléments nouveaux après l'autobiographie de Lovelock. Les éléments qui suivent sont également basés sur une exploitation des archives de Lovelock, un entretien avec Lovelock [Sébastien Dutreuil, avril 2016] et des entretiens avec les deux étudiants de Lovelock: Timothy Lenton et Andrew Watson [Sébastien Dutreuil, janvier à avril 2014].

39 James Lovelock, O. M. Lidwell, et W. F. Raymond " Aerial disinfection. ", Nature, vol. 153, $\mathrm{n}^{\circ}$ 3870, 1944, p 20-21; James Lovelock, O. M. Lidwell, et W. F. Raymond « Vaporization of lactic acid as an aerial bactericide. " Nature, vol. 153, n 3894, 1944, p. 743-743 ; James Lovelock, "Aerial bactericides ", Nature, vol. 156, $\mathrm{n}^{\circ} 3974,1945, \mathrm{p} .782-783$.

40 James Lovelock et J. S. Porterfield, « Blood coagulation : its prolongation in vessels with negatively charged surfaces. ", Nature, vol. 167, n 4236, 1951, p. 39-40.

41 James Lovelock " The haemolysis of human red blood-cells by freezing and thawing ", Biochimica et biophysica acta, $\mathrm{n}^{\circ} 10,1953$, p. 414-426; James Lovelock, " The physical instability of human red blood cells», Biochemical Journal, vol. 60, $\mathrm{n}^{\circ}$ 4, 1955, p. 692-696 ; James Lovelock et M. W. H. Bishop " Prevention of freezing damage to living cells by dimethyl sulphoxide. » Nature, vol. $183, \mathrm{n}^{\circ} 4672,1959, \mathrm{p}$. 1394-1395.

42 A. U. Smith, James Lovelock, et A. S. Parkes « Resuscitation of Hamsters after Supercooling or Partial Crystallization at Body Temperatures Below $0^{\circ} \mathrm{C}$. », Nature, vol. 173, n 4415, 1954, p. 1136-1137.

43 James Lovelock, " Affinity of organic compounds for free electrons with thermal energy : its possible significance in biology ", Nature, vol. 189, $\mathrm{n}^{\circ}$ 4768, 1961, p. 729-732 ; James Lovelock, P.G. Simmonds, et W.J. Vandenheuvel « Affinity of steroids for electrons with thermal energies. " Nature, vol. 197, $\mathrm{n}^{\circ} 4864$, 1963, p. 249-251.

${ }^{44}$ Sébastien Dutreuil, « James Lovelock's Gaia hypothesis : 'a new lookt at life on Earth' ... for the life and the Earth sciences ", in Michael Dietrich et Oren Harman, Dreamers, romantics and visionaries in the life sciences, Chicago, Chicago University Press, 2017. 
Commoner ${ }^{45}$. Son goût, comme ingénieur, pour les circuits électroniques, l'a familiarisé avec la cybernétique : c'est précisément la cybernétique de premier ordre, celle des feedbacks, des thermostats, et des circuits électroniques qui est mise en avant comme cadre théorique pour rendre compte des phénomènes de régulation de Gaïa ${ }^{46}$. Et si Gaïa est comparée à un vivant, elle est aussi facilement et sans transition comparée au thermostat d'un four. Enfin, son statut d'ingénieur dans le domaine de la chimie l'a placé au centre d'un réseau dense de relations scientifiques et industrielles, décisives pour l'élaboration de Gaïa (cf. infra).

\section{La collaboration avec Margulis}

Le premier texte publié comportant le nom de Gaïa est de Lovelock en $1972^{47}$. Et les première formulations d'une hypothèse qui ressemble à celle qui sera nommée " hypothèse Gaïa » par Lovelock et Margulis en $1974^{48}$ sont publiées dans le compte-rendu d'une conférence de Lovelock et Giffin en $1969^{49}$. Si Lovelock avait les premiers éléments sur Gaïa avant de rencontrer Margulis à la fin des années 1960, il reste que Margulis a eu un rôle décisif dans I'histoire d'HG.

Cette citation illustre de manière rétrospective les complémentarités entre Margulis et Lovelock :

La conclusion est inévitable : les géophysiciens et les scientifiques de l'atmosphère doivent étudier la biologie et les biologistes doivent savoir quelque chose de la géophysique et de la science de l'atmosphère. Pendant trop longtemps, nous avons des chimistes de l'atmosphère se demandant ' $D$ 'où vient tout ce méthane?', et des biologistes ignorant 'où tout ce méthane va'50.

Cette remarque ne manque pas de saveur lorsque l'on sait que Lovelock et Margulis correspondaient, au début des années 1970, exactement aux deux personnages abstraits critiqués dans cette citation: Lovelock s'intéressait alors à la chimie de l'atmosphère et demandait à Margulis son aide pour identifier, dans le monde vivant, des sources de méthane et d'autres composés chimiques. Margulis n'a pas seulement apporté à HG ses connaissances en écologie microbienne, mais également un réseau scientifique complémentaire de celui de Lovelock. Basée aux États-Unis tandis qu'il était en Angleterre, les deux scientifiques échangeaient souvent leurs vues respectives sur l'état des sciences dans leurs propres pays et sur la réception de Gaïa. En outre, c'est également elle qui avait initialement, dans les années 1970, attiré l'attention de Lovelock vers les questions et les acteurs de l'histoire de la Terre et de la vie, des champs qui étaient auparavant étrangers à Lovelock. Celui-ci se concentrait sur la chimie et le climat contemporains mais connaissait mal les travaux en paléontologie et ceux

\footnotetext{
45 Sur l'importance des sciences de la Terre et des sciences physiques de l'environnement - relativement à l'écologie - pour l'émergence des questions environnementales, voir : Ronald E. Doel, « Quelle place pour les sciences de l'environnement physique dans l'histoire environnementale? ", Revue d'histoire moderne et contemporaine, vol. 56, $n^{\circ} 4$, 2009, p. 137-164.

46 Sur l'histoire de la cybernétique, voir les études de : Peter Galison, "The ontology of the enemy: Norbert wiener and the cybernetic vision ", Critical inquiry, vol. 21, n 1, 1994, p. 228-266 ; Céline Lafontaine, L'empire cybernétique: des machines à penser à la pensée machine, Paris, Seuil, 2004 ; Andrew Pickering, The cybernetic brain : Sketches of another future, Chicago, University of Chicago Press, 2010. On peut écrire une histoire parallèle de la cybernétique et d'HG : de Lovelock et la cybernétique de premier ordre à Lenton puisant dans les sciences de la complexité en 1990, en passant par Margulis et la cybernétique de second ordre de Varela dans les années 1970/1980 - voir Dutreuil, Gaïa. Op. cit.

47 Lovelock, " Gaia as seen ». Op. cit.

48 Lovelock et Margulis, "Atmospheric homeostasis ». Op. cit.

49 James Lovelock et C.E. Giffin, « Planety atmospheres : Compositional and other changes associated with the presence of life.», Advances in the Astronautical Sciences, vol. 25, 1969, p. 179-193.

50 Lynn Margulis et Gregory Hinkle, "The biota and Gaia : 150 years of support for environmental sciences. ", in Stephen H. Schneider et Penelope J. Boston, Scientists on Gaia, Cambridge, MA, The MIT Press, 1991, p. 12.
} 
des géochimistes étudiant l'histoire chimique de la Terre dans la longue durée - deux champs avec lesquels Lovelock et Margulis vont initier des interactions, notamment à partir de la fin des années 1970.

Les complémentarités initiales de Lovelock et Margulis ont ainsi rendu possible une diffusion très large d'HG dans des cadres disciplinaires relativement séparés: la climatologie contemporaine, la chimie de l'atmosphère, l'histoire de la Terre (géochimie et paléoclimatologie), l'écologie, ou encore les sciences de la complexité. Après le début des années 1980, ils ne publient que très rarement ensemble ${ }^{51}$.

\section{Lovelock : scientifique indépendant et solitaire ou scientifique entrepreneur au réseau étendu?}

Lovelock quitte le monde académique à partir de 1964 et tire profit de ce retrait en se présentant constamment comme un scientifique indépendant et solitaire, travaillant seul dans le laboratoire qu'il s'est constitué dans son garage, au cœur de la campagne du sud-ouest de l'Angleterre. C'est le titre de son autobiographie : Hommage à Gaïa : la vie d'un scientifique indépendant.

Cette rhétorique de l'indépendance mêle deux aspects. Le premier se situe sur un registre épistémologique et a trait au métier même de scientifique. Pour Lovelock, la science doit être l'affaire de quelques scientifiques géniaux et inspirés, plutôt que d'une armée de spécialistes, d'experts et de fonctionnaires de la recherche. Les textes de Lovelock sont ainsi émaillés de propos qui l'habillent avec un ethos d'artiste solitaire, évoquant la figure du scientifique romantique du $19^{\mathrm{e}}$ siècle. Voici par exemple ce qu'il dit à propos d'une de ses rencontres :

« Il fut un modèle pour moi du scientifique solitaire, celui qui, comme l'artiste ou le romancier, produit le meilleur de son travail créatif seul. „152

La figure alterne entre celle de l'artiste, du créateur et de l'inventeur :

"La composition de musique, l'écriture de vers et de romans, la peinture d'images et la sculpture de statues, toutes sont reconnues et louées comme créatives, mais considérez une roue ou une goupille de sécurité : ce ne sont pas vraiment des travaux d'art mais ce sont sans aucun doute des créations et de bonnes inventions, alors pourquoi, en Angleterre, quoique cela ne soit pas le cas en Écosse, nous regardons ces inventions comme inférieures ? ${ }^{53}$

Pour comprendre la position de Lovelock dans les années 1960 et 1970, et tempérer son caractère iconoclaste, il est nécessaire de revenir sur des transformations importantes du métier de scientifique. L'histoire de ces transformations, retracée dans un livre remarquable de Steven Shapin, va du génie solitaire et exceptionnel dont la vocation est inspirée par Dieu au $17^{\mathrm{e}}$ et $18^{\mathrm{e}}$ siècle à l'homme ou la femme ordinaire, employé(e) d'un État et participant à une entreprise collective au $20^{\mathrm{e}}$ siècle en passant par la figure de l'amateur au $19^{\mathrm{e}}$ siècle. Dans les années 1970, cette histoire est marquée par un tournant important, avec l'émergence de la figure du scientifique entrepreneur :

«L'entrepreneur scientifique est défini comme celui qui est à la fois un scientifique qualifié et, comme tous les entrepreneurs commerciaux, quelqu'un qui prend des risques. (...) Ils ont un pied dans la production de connaissances et l'autre dans la fabrications d'instruments, de services et, finalement d'argent. Ils peuvent ou non être conscient de l'existence de 'conflits' entre ces

\footnotetext{
${ }^{51}$ Notamment après que Lovelock ait commencé à développer le modèle Daisyworld pour soutenir HG, Margulis rejetant toute forme d'argumentation basée sur des modèles mathématiques. Margulis s'est en outre concentrée sur le développement de ses conceptions sur la symbiose, au sein desquelles Gaïa constituait un chapitre plutôt que l'armature centrale.

52 Lovelock, Homage. Op. cit., p. 78.

53 lbid., p. 176
} 
aspects de leur identité, mais ils incarnent des conduites et des activités qui, au cours du vingtième siècle, ont largement été considérées comme en tension, et parfois en opposition. ${ }^{54}$

Les exemples typiques de scientifique entrepreneur de Shapin participent à l'essor des start-ups de biotech dans la Silicon Valley. Ses acteurs n'hésitent pas à mettre en avant la valeur de l'argent : le slogan de l'époque était « s'éclater et faire de l'argent ».

Contrairement aux entrepreneurs de Shapin, Lovelock n'a jamais explicitement mis en avant la valeur de l'argent et lui a toujours préféré la liberté et la créativité scientifique. Il cultive plus volontiers un isolement à la campagne que la plongée dans un réseau social riche et dense. Mais pour le reste, la situation professionnelle de Lovelock au moment même de l'élaboration de Gaïa correspond parfaitement au portrait de l'entrepreneur scientifique que dresse Shapin. Lovelock n'a ainsi jamais caché son mépris pour la figure scientifique à laquelle s'oppose celle de l'entrepreneur : le scientifique fonctionnaire. II fait même des émules dans son entourage ${ }^{55}$.

Le second aspect concernant le thème du scientifique solitaire et indépendant porte moins sur la question épistémologique de l'organisation du travail scientifique et de l'ethos du savant, mais plus directement sur la question de l'indépendance financière.

\section{Le détecteur à capture d'électron au centre des activités et du réseau de Lovelock}

Si Lovelock a ressenti le besoin d'écrire très tôt, dans les colonnes de New Scientist, un texte sur son statut de "scientifique indépendan ${ }^{56}$, c'est bien parce que le reproche lui était fait d'être dans une situation de possible conflit d'intérêt, notamment dans le cadre de la controverse sur l'interdiction des $\mathrm{CFCs}^{57}$. Lovelock est l'inventeur de nombreux instruments et détenteur de plusieurs brevets. L'instrument pour lequel il est resté le plus célèbre est sans conteste l'ECD, le détecteur à capture d'électrons, qu'il a inventé en 1957. L'ECD permettait des mesures de composés chimiques d'une sensibilité supérieure de plusieurs ordres de grandeur aux instruments de l'époque. Cette invention a permis à Lovelock de s'établir comme consultant scientifique. À partir du début des années 1960, Lovelock devient ainsi progressivement consultant pour des grandes entreprises chimiques et pétrolières - parmi lesquelles Shell, Hewlett Packard, Imperical Chemical Industries (la plus grande industrie chimique et plus grosse productrice de CFCs en Grande-Bretagne), DuPont de manière ponctuelle -, mais aussi le Ministère de la Défense Britannique et les services secrets (le MI6), ou encore des institutions scientifiques comme les agences américaines de l'espace et de l'étude du climat et des océans - la NASA puis la NOAA (National Oceanic and Atmospheric Administration). Pour ses travaux Lovelock facture ses services à la journée et passe de l'un à l'autre au gré des commandes. II gagne confortablement sa vie. Lord Rotschild, son employeur à Shell, est ainsi surpris d'apprendre que le salaire total de Lovelock dépasse celui des directeurs de Shell ${ }^{58}$.

C'est parce que Lovelock menait ces travaux de consultant qu'on lui a reproché sa position dans la controverse sur l'ozone dans les années 1970 (Lovelock s'opposait à une interdiction immédiate des CFCs et arguait que le temps pour légiférer n'était pas encore venu), et

\footnotetext{
54 Erreur! Source du renvoi introuvable., p. 210.

55 Ainsi, dans les années 1970, un chimiste proche de Lovelock, auparavant au NCAR (National Center For Atmospheric Research, Boulder, Colorado) lui écrit pour lui dire qu'il l'a pris comme modèle pour s'établir comme " consultant indépendant » [Lettre de James Lodge à James Lovelock, 15 juillet 1974, archives de Lovelock].

56 James Lovelock, "The independent practice of science. ", New Scientist, September 6, 1979, p. 714-717.

57 Je n'ai pas trouvé de traces écrites de tels reproches, mais, au cœur des années 1970, ces reproches étaient souvent fait oralement, lors de conférences [entretien avec Andrew Watson, Sébastien Dutreuil, février 2017].

58 Lovelock, Homage. Op. cit., p. 158.
} 
notamment son témoignage devant le Congrès américain, en faveur de DuPont ${ }^{59}$. Si Lovelock a été un acteur de premier plan dans la controverse sur l'ozone, c'est précisément parce qu'il avait été le premier à mesurer la concentration en CFCs dans l'atmosphère, grâce à l'ECD ${ }^{60}$, avant qu'un modèle ne suggère que ces composés pourraient détruire l'ozone stratosphérique ${ }^{61}$.

La NOAA avait fait appel à Lovelock dans les années 1970 pour mettre au point une méthode permettant de suivre les masses d'air. Voici ce que dit Lester Machta, directeur de la NOAA Air Resources Laboratory (et de l'institution qui la précédait) de 1948 à 1989, à propos de ce travail de Lovelock :

" Jim était un très bon ami à nous - le mien en particulier. (...) Comme vous le savez, il a inventé la chromatographie à capture d'électrons qui permettait de voir les CFC et le méthane (...). S'il y avait un seul bénéfice que nous ayons eu de l'extérieur, mon avis est que mis à part Dave Keeling nous ayant montré comment faire des mesures de $\mathrm{CO}_{2}$, il est venu de Jim Lovelock. II a été très, très utile pour nous. " ${ }^{62}$

Le travail d'ingénieur de Lovelock de fabrication et de mise au point d'instruments, de conseil et de veille scientifique, auprès des institutions importantes pour la climatologie et l'océanographie, a ainsi joué un rôle tout à fait significatif dans l'acceptation et l'accueil de ses vues plus spéculatives sur Gaïa. Pour ne donner qu'un exemple, en 1985, Lovelock est invité pour faire un séminaire exceptionnel pour célébrer les 25 ans du NCAR, le National Center for Atmospheric Research, temple de la climatologie américaine et mondiale. Voici un extrait de la lettre d'invitation qu'il reçoit de la part de l'institution :

« Nous sommes de plus en plus forcés, dans nos études, à considérer le couplage de l'atmosphère avec non seulement d'autres systèmes géophysiques évidents, tels que les océans et les calottes glaciaires mais aussi avec le changement solaire, la dérive des continents, et la biosphère. Nous pensons donc qu'une discussion de ces couplages, en particulier avec la biosphère, constitue un élément important de tout programme examinant le futur de notre science.

Ton travail sur les techniques de mesure des gaz présents en traces est bien connu des chimistes de l'atmosphère ici, et nous nous rappelons de certaines de tes contributions dans ce domaine à chaque fois qu'il y a une résurgence d'intérêt pour les CFCs dans l'atmosphère. (...) Mais évidemment, la raison pour laquelle nous voulons que tu viennes et parles est plus large : nous voulons nous maintenir au fait de tes perspectives et spéculations à propos de la nature et du futur des études du système biosphère-atmosphère.. " ${ }^{63}$

L'expertise internationalement reconnue - et, dans les années 1960 et 1970, littéralement unique au monde - de Lovelock pour la mesure de quantités infimes de composés chimiques, et donc ses contributions précises et détaillées en chimie de l'atmosphère, ont ainsi constitué une porte d'entrée remarquable pour Gaïa en sciences de l'atmosphère et de la Terre.

${ }^{59}$ Sur la controverse de l'ozone voir les textes classiques de : Oreskes et Conway, The Merchants. Op. cit. ; Grevsmühl, La Terre, Op. cit. - et en particulier la belle thèse de Régis Briday, très détaillée sur cette question: Régis Briday, Une histoire de la chimie atmosphérique globale. Enjeux disciplinaires et d'expertise de la couche d'ozone et du changement climatique, Thèse, Centre Alexandre Koyré, 2014. Nous revenons également sur cette question dans notre thèse : Dutreuil, Gaïa. Op. cit.

60 James Lovelock, R. J. Maggs, et R. J. Wade, " Halogenated hydrocarbons in and over the atlantic ", Nature, vol. 241, $\mathrm{n}^{\circ}$ 194-196, 1973.

${ }^{61}$ Mario J. Molina et F. Sherwood Rowland, "Stratospheric sink for chlorofluoromethanes : chlorine atom-catalysed destruction of ozone. ", Nature, vol. 249, n 5460, 1974, p. 810-812.

${ }^{62}$ Lester Machta, entretien avec Spencer Weart et William Elliott, American Institute of Physics, Oral history Interviews : Lester Machta (interview : 25.04.1991), 1991.

63 La lettre, du 16 mai 1985 [archives de Lovelock], n'est malheureusement pas signée, seul l'en-tête du NCAR figure. Elle émane sans doute de Walter Roberts, ou de Wilmot Hess, alors directeur du NCAR. 
Qu'il faille inventer un dispositif de mesure pour repérer les fuites d'un pipeline, suivre à la trace une masse d'air pour déterminer le trajet des pollutions au-dessus des grandes villes ou pour affiner notre compréhension de la dynamique de l'atmosphère, ou bien mesurer les concentrations de composés chimiques à travers les océans et l'atmosphère, dans les années 1960 et 1970, c'est Lovelock qu'il vous faut. Dans les décennies de l'élaboration d'HG, les travaux de Lovelock, développés en Angleterre, en Irlande et aux États-Unis, se situent donc au carrefour de problèmes techniques des industries chimiques et des sciences étudiant les pollutions de l'environnement global, de la climatologie à la chimie de l'atmosphère, en passant par l'océanographie.

\section{Qu'est-ce que Gaïa? Un cadre pour penser les problèmes de pollution globale}

Ces éléments nous permettent d'apporter un éclairage nouveau sur l'histoire de Gaïa. Lovelock a souvent mis en avant l'importance de réflexions spéculatives à la NASA sur des critères de détection de la vie sur d'autres planètes comme étant à l'origine d'HG. C'est en cherchant un tel critère que Lovelock aurait reconnu l'influence massive qu'ont les vivants sur l'atmosphère terrestre, puis proposé que le critère repose sur la reconnaissance d'un déséquilibre thermodynamique dans l'atmosphère des planètes ${ }^{64}$ et enfin formulé l'hypothèse suivant laquelle les vivants pourraient, via leur influence, réguler l'environnement ${ }^{65}$.

Cette histoire, sans être dénuée de fondements, passe entièrement à côté de la matrice dense des travaux de Lovelock au moment de l'élaboration d'HG, entre 1965 et 1979. Pendant cette période, Lovelock ne travaille plus pour la NASA et n'est plus occupé à réfléchir et publier des textes d'exobiologie. Le cœur des activités quotidiennes de Lovelock porte sur des questions de pollution.

Rappelons la thèse historique centrale que nous proposons: si on veut comprendre ce qu'est Gaïa et sa trajectoire historique, il faut d'abord et avant tout la voir comme l'élaboration d'un nouveau cadre général pour penser le phénomène de pollution globale, et non comme une réflexion spéculative et abstraite sur la nature de la vie ou la biologie évolutive.

\section{La première formulation de l'hypothèse Gaïa : une note prospective pour Shell}

Si la première formulation publiée d'éléments très proches de ce qui s'appellera dans les années 1970 « hypothèse Gaïa » date de 196966, la première trace écrite remonte elle à 1966. La nature du document est instructif : il s'agit d'une note prospective que Lovelock écrit pour Shell. La note de novembre 1966 est intitulée "Remarques sur l'année 2000 " [Archives de Lovelock]. Elle se démarque d'une note publiée par ESSO, qui table sur une croissance économique stable à $5 \%$, que Lovelock a lue et dont il veut prendre le contrepied. Après des remarques liminaires sur la difficulté d'un exercice prospectif, Lovelock commence par une longue page sur l'importance de la croissance de la population et sur la manière dont ce facteur, inconnu, conditionne et déterminera l'avenir - nous sommes deux ans avant La bombe $P^{68}$ et 6 ans avant le rapport du Club de Rome sur les limites à la croissance. II poursuit en indiquant que le danger le plus important pour la croissance à venir sera une forme de pollution, résultant de l'accumulation d'un déchet. II indique qu'il existe déjà des signes que la pollution de l'air est devenu un problème global et pourrait se développer de manière catastrophique dans moins de dix ans. II prédit en conséquence des changements drastiques des modes de déplacement, de consommation d'énergie et d'utilisation des terres ainsi que le déploiement de modes de communication par satellites. La dernière prédiction est une " surprise désagréable » : un âge glaciaire dans les prochaines décennies.

\footnotetext{
64 Lovelock, "A physical ». Op. cit.

65 Lovelock et Margulis, "Atmospheric homeostasis ». Op. cit.

66 Lovelock et Giffin, «Planetary ». Op. Cit.

67 Archives de Lovelock.

68 Paul Ehrlich, The population bomb, New York, Ballantine Books, 1968.
} 
Des prospectives pour Shell suivent. Lovelock prédit un avenir radieux à Shell qui pourra jouer un rôle important en :

- Bitumant les surfaces désertiques pour diminuer l'albédo de la Terre (et contrer le refroidissement à venir) ;

- Modifiant délibérément la composition de l'atmosphère en injectant un composé spécifiquement choisi pour ses propriétés radiatives (de gaz à effet de serre).

Rédigée 40 ans avant le livre polémique La revanche de Gaïa, cette note en rappelle l'essentiel des thématiques, des problèmes à cibler en priorité, et des solutions proposées, le trio: problèmes environnementaux globaux (climat et pollution), surpopulation, solutions techniques proches de la géoingénierie. Voici comment elle s'achève :

" Au cours des 33 prochaines années, nous entrons dans une phase où l'activité humaine devient une partie significative de l'activité biologique totale de la planète. Jusqu'alors le climat de la Terre et la composition chimique de la surface, de l'air et de la mer ont évolué avec la vie pour fournir des conditions optimum pour sa survie; de plus, cet optimum était activement maintenu par des processus biologiques cybernétiques. C'est dans l'intérêt de Shell que de participer au maintien de cet optimum quelle que soit la cause d'un écart à celui-ci. Cela devrait être un défi intéressant et nous maintenir en vie. " ${ }^{69}$

C'est donc dans la conclusion de cette note prospective pour Shell que l'on trouve une première trace écrite de propositions très proches de ce qui sera appelé HG.

\section{La pollution : le cœur de métier de Lovelock au moment de l'élaboration de Gaïa}

Plus largement, pour ainsi dire tous les travaux de Lovelock au moment de l'élaboration d'HG portent sur des questions de pollution. Nombre de ses articles spécialisés portant directement sur des questions de pollution font appel, à un moment de l'argumentation, à l'idée gaïenne suivant laquelle l'environnement est régulé par la vie ; et réciproquement, ses textes portant sur Gaïa portent toujours la marque de ses travaux sur la pollution. Donnons quelques exemples.

L'article de Lovelock et Giffin de $1969^{70}$ s'achève ainsi sur les conséquences de ces réflexions pour penser les problèmes écologiques de dimension globale, comme la manière dont la combustion d'énergies fossiles pourrait influencer la composition globale en oxygène. En 1971 l'article qu'il publie dans la revue Atmospheric Environment, intitulé " Air pollution and climatic change " dont l'objectif est d'évaluer les possibilités de changements globaux en conséquence d'émissions de combustibles, développe la discussion en partant de "la prémisse que l'atmosphère est un système biologique cybernétique et que les combustions constituent une perturbation de ce système ", rappelant l'idée centrale $\mathrm{d}^{\prime} \mathrm{HG}^{71}$. Avant d'être une hypothèse à tester, Gaïa était déjà un cadre pour penser les perturbations anthropiques sur un système atmosphérique et biologique.

Une idée phare d'HG, publiée en 1987 et connue comme l'hypothèse CLAW, suivant les initiales des auteurs de l'article, est l'idée que les algues pourraient contribuer à réguler le climat via l'émission d'un composé soufré, le $\mathrm{DMS}^{72}$. Cet article a été cité plusieurs milliers de fois et l'influence du DMS sur le climat est encore une source d'incertitude majeure sur l'évolution à venir du climat. Les travaux de Lovelock sur le DMS remontent à des mesures qu'il

\footnotetext{
69 Lovelock, "Some thoughts on the year 2,000 », archives de Lovelock.

70 «Planetary ». Op. Cit.

71 James Lovelock, " Air pollution and climatic change. ", Atmospheric Environment, vol. 5, n 6, 1971, $\mathrm{p}$. 403.

72 Robert Charlson, James Lovelock, Meinrat Andreae, et Stephen Warren, « Oceanic phytoplankton, atmospheric sulphur, cloud albedo and climate ", Nature, vol. 326, n 6114, 1987, p. 655-661.
} 
a effectuées dans les années 1970 de ce composé ${ }^{73}$. Rétrospectivement, Lovelock présente ce texte comme le fruit d'une recherche gaïenne portant sur la manière dont la vie peut contribuer aux grands cycles biogéochimiques et à la circulation de la matière terrestre ${ }^{74}$. $\mathrm{S}^{\prime} \mathrm{il}$ est bien question de réflexions sur le bouclage du cycle du soufre dans l'article de 1972, on ne trouve pas de réflexions sur HG. En revanche, cet article, dont Lovelock et l'un des deux co-auteurs sont financés par Shell, se clôt sur l'idée que certains produits dérivés du DMS, ayant des propriétés proches de l'acide sulfurique, ont une durée de vie importante dans l'atmosphère ${ }^{75}$. L'acide sulfurique est issu de la réduction du dioxyde de soufre contenu dans les combustibles fossiles. Dans les années 1960, on s'inquiétait que ces émissions issues des combustibles fossiles contribuent à refroidir le climat et à déclencher un nouvel âge glaciaire Lovelock avait écrit des rapports pour Shell et abondamment échangé avec Lord Rotschild sur cette question brûlante. Les mesures de DMS s'insèrent donc au moins autant dans des réflexions urgentes pour une industrie pétrolière émettrice d'oxydes de soufre qu'elles prennent leur place dans un programme de recherche sur l'économie de la nature et ses grands cycles gaïens.

La diversité de ses clients a eu pour Lovelock une conséquence décisive. II s'est retrouvé être tantôt le témoin indirect et parfois direct, tantôt l'acteur de premier plan pour la totalité des problèmes émergents de pollution de l'environnement global classiquement liés à l'émergence des mouvements environnementalistes pendant cette période : les conséquences dévastatrices du DDT révélées par Carson dans les années 1960 (le DDT est un insecticide organo-chloré dont Shell était un producteur important, et dont les quantités traces ont pu être mesurées partout à la surface du globe grâce ... à l'ECD inventé par Lovelock); l'émergence d'une destruction de la couche d'ozone dans les années 1970 et 1980 (résultant de l'accumulation de CFCs produits par Dupont ou Imperical Chemical Industries); le changement climatique dès les années 1960 (les combustibles fossiles étaient déjà mis en cause) ; les pluies acides dans les années 1970 et 1980 (conséquence de la libération d'oxydes de soufre par les mêmes combustibles fossiles). Lovelock est également directement impliqué dans d'autres problèmes moins connus comme les nitrates de péroxyacétyles (un des composants des smogs urbains). Sa position de consultant pour Shell l'amène même à croiser le problème de la pollution au plomb par l'essence, avant que l'essence sans plomb ne soit généralisée.

Il s'agit moins pour nous ici de dénoncer d'éventuels conflits d'intérêt entre les positions de Lovelock et ses activités de consultant que de souligner le fait historique décisif mais insuffisamment remarqué suivant lequel la préoccupation majeure et quotidienne de Lovelock dans les décennies d'élaboration de Gaïa est celle de la pollution de l'environnement global, sous toutes ses formes connues.

\section{Comprendre les pescriptions de Lovelock : naturalisation de la pollution et géophysiologie gaïenne}

Il est dès lors utile d'essayer d'analyser le rapport que l'élaboration de Gaïa comme cadre de pensé a entretenu avec les prises de positions politiques de Lovelock sur des questions environnementales. Qu'est-ce qui fonde les prescriptions de Lovelock et quelle articulation existe-t-il entre sa conception de Gaïa et ses prescriptions ?

\section{Une critique classique : polluez, Gaïa régulera}

Le lien le plus visible, et le seul jusque-là commenté, est celui qui existe entre le caractère " homéostatique » et " robuste » de Gaïa, et la prise en charge des perturbations anthropiques par Gaïa: polluez, Gaïa régulera. Cette lecture est juste au premier ordre. C'est celle de

\footnotetext{
73 James Lovelock, R. J. Maggs, et R. A. Rasmussen « Atmospheric dimethyl sulphide and the natural sulphur cycle », Nature, vol. 237, 1972, p. 452-453.

74 James Lovelock, Gaia : medicine for an ailing planet, London, Gaia Books, 2005 (1991), p. 122-123.

75 Lovelock, Maggs, et al., « Atmospheric ». Op. cit., p. 453.
} 
nombreux scientifiques ayant pris part au débat sur HG. C'est ainsi l'inquiétude qui avait été très tôt exprimée par les participants d'une conférence importante en climatologie, organisée entre autres par William Kellog, Margaret Mead, Walter Orr Robert et Lovelock en 1975 et au cours de laquelle Lovelock avait exposé ses vues sur Gaïa. Plus tard, Crutzen souligne ainsi que les conceptions de Gaïa qu'avait Lovelock ne sont certainement pas pour rien dans la sous-estimation systématique des problèmes environnementaux de Lovelock dans les années 1970 et en particulier pour ce qui concerne le danger des $\mathrm{CFCs}^{76}$.

Cette lecture peut être étayée par des citations de Lovelock dans les années 1970. Pour ne donner qu'un exemple, après avoir présenté les conséquences désastreuses de l'oxygénation de l'atmosphère il y a plus de 2 milliards d'années, voici ce qu'écrivaient Lovelock et Sydney Epton (chercheur à Shell) dans cet article de 1975 paru dans New Scientist :

"L'activité présente de l'Homme en tant que pollueur est triviale en comparaison et par conséquent il ne peut pas sérieusement changer l'état présent de Gaïa, encore moins mettre en danger son existence. ${ }^{77}$

Mais il est possible d'analyser plus en finesse les liens entre Gaïa et les prescriptions politiques de Lovelock: celles-ci reposent toutes sur la même structure argumentative. Le premier temps vise à naturaliser le concept de pollution. Le second souligne bien que Gaïa ne pourra pas absorber toutes les perturbations en restant stable et robuste. Mais les activités humaines susceptibles de perturber Gaïa ont pour Lovelock toujours la même source : non pas l'essor de l'industrie chimique, mais la surpopulation, médiée par les effets néfastes de l'agriculture. Détaillons.

\section{Un argument plus subtil $1 / 2$ : naturaliser la pollution}

Si les humains font partie de Gaïa, alors les actions des humains sont naturelles ou gaïennes, y compris celles classiquement considérées comme des pollutions :

" Suivant l'aliénation persistante à la nature que nous nous imposons, nous avons tendance à considérer que nos produits industriels ne sont pas 'naturels'. En fait, ils sont autant naturels que le sont les autres produits chimiques de la Terre, car ils ont été produits par nous, qui sommes assurément des créatures vivantes. Ils peuvent bien sûr être agressifs et dangereux, comme les gaz neurotoxiques, mais ils ne le sont pas davantage que les toxines fabriquées par botulinus bacilluls. ${ }^{78}$

La question de savoir ce que constitue une pollution la question qui préoccupe Lovelock de manière centrale, notamment dans son premier livre sur Gaïa ${ }^{79}$. La thèse qu'il défend est que le concept de pollution, conçu comme dégradation intrinsèquement anthropique de l'environnement, est un concept problématique :

"Se pourrait-il que la pollution soit naturelle ? (...) Le concept même de pollution est anthropocentrique et il peut être non pertinent dans un contexte Gaïen. » ${ }^{80}$

Inlassablement, Lovelock argumente en faveur d'une naturalisation du concept de pollution. Les produits chimiques industriels sont considérés comme des poisons; mais le plus grand épisode de pollution de la planète n'est-il pas l'oxygénation de l'atmosphère ayant eu lieu il y a

\footnotetext{
76 Paul J. Crutzen, « A critical analysis of the gaia hypothesis as a model for climate/biosphere interactions ", Gaia, vol. 11, n², 2002, p. 102.

77 James Lovelock et Sidney Epton, "The quest for gaia », New Scientist, 6 février 1975, p. 305. Voir également : James Lovelock, Gaia: A new look at life on Earth, Oxford, Oxford University Press, 1979, p. 109.

78 Ibid., p. 75 (voir également p. 101-103).

79 Ibid.

80 lbid, p. 108, 110.
} 
plusieurs milliards d'années, dont les responsables sont bien des plantes ${ }^{81}$ ? Les CFCs contribuent à apporter du chlore dans la stratosphère et ainsi à détruire l'ozone stratosphérique; mais les volcans, ainsi que d'autres composés naturels ou biologiques peuvent de la même manière apporter des composés chlorés dans la stratosphère ${ }^{82}$.

$\mathrm{Si}$ des organismes produisent un composé et affectent ainsi l'environnement, cette production peut dès lors être interprétée de manière fonctionnelle : la destruction partielle de l'ozone (compensant potentiellement d'autres mécanismes augmentant la production d'ozone) pourrait ne pas être que néfaste. Elle pourrait avoir un rôle important dans Gaïa pour le maintien de certains équilibres.

Et les vivants ne sont-ils pas capables en retour de s'adapter à ces épisodes de pollution, comme la phalène du bouleau s'est adaptée aux conséquences des activités industrielles du $19^{e}$ siècle ${ }^{83}$ ? De même que les vivants se sont adaptés à une atmosphère oxygénée, de même pour Lovelock, les humains à devraient s'adapter à une augmentation de l'intensité des UVs résultant de l'amincissement de la couche d'ozone, si des tabous raciaux n'empêchent pas de flux génétiques entre les populations les plus sensibles aux UV et celles qui y sont plus adaptées ${ }^{84}$.

Non seulement les vivants s'adaptent, mais les problèmes de pollution sont systématiquement résolus par la sélection naturelle lorsqu'un organisme apparaît qui se nourrit de cette "pollution ${ }^{85}$. Et voilà in fine la pollution connotée de manière positive. La pollution c'est non seulement inévitable et naturel (et on s'y adapte), mais la pollution c'est également bon pour les vivants :

"La pollution n'est pas, comme on le dit trop souvent, le produit d'une turpitude morale. C'est la conséquence inévitable de la vie à l'œuvre. Pour l'herbe, les scarabées et même les fermiers, la bouse de vache n'est pas une pollution mais un don précieux. Dans un monde raisonnable, les déchets industriels ne seraient pas interdits mais utilisés à bon escient. La réponse négative, non constructive, de l'interdiction par la loi semble aussi idiote que de légiférer contre l'émission de bouses par les vaches. ${ }^{86}$

On a souvent célébré Gaïa comme une forme de réenchantement de la nature : après des siècles de séparation entre les humains et la nature, Gaïa est l'occasion d'un grand retour à une conception harmonieuse plaçant l'homme en continuité avec la nature et son environnement. Le revers de la médaille, et, en fait, ce qui constitue sans doute une des origines importantes de Gaïa, est que les actions humaines ne justifient plus d'un caractère d'exception sur le plan moral et ne peuvent plus être dénoncées comme telles : la pollution devient un fait naturel et doit être analysée comme telle.

81 Ibid., p. 109

82 Lovelock, Maggs, et al., " Halogenated ». Op. cit., p. 195-196 ; James Lovelock, " Natural halocarbons in the air and in the sea. ", Nature, vol. 256, 1975, p. 193-194 ; Lovelock, Gaia. Op. cit., p. 115.

83 Lovelock, « Air pollution ». Op. cit., p. 410 ; James Lovelock et Lynn Margulis « Homeostatic tendencies of the earth's atmosphere. ", Origins of Life and Evolution of Biospheres, vol. 5, $n^{\circ} 1,1974$, p. 102 ; Lovelock, Gaia. Op. cit., p. 109, 110.

84 Ibid., p. 110-111.

85 Incidemment, soulignons que les rares références à la sélection naturelle dans le premier livre de Lovelock sur Gaïa sont associées à cette idée suivant laquelle la sélection permet de "régler » les problèmes de pollution en faisant disparaître les vivants qui ne sont pas adaptés ou en permettant l'émergence d'une innovation évolutive contre-balançant les pollutions précédentes.

86 Ibid., p. 27-28. Voir également : James Lovelock, The ages of Gaia : a biography of our living earth, Oxford, Oxford University Press, second edition, 1995 (1988), p. 26. 


\section{Un argument plus subtil (2/2) - Le coupable : non pas l'industrie chimique mais l'agriculture}

Du début des années 1970 à la fin des années 1980, la naturalisation de la pollution s'accompagne toujours chez Lovelock d'une rhétorique visant à orienter le regard accusateur dans une direction privilégiée : il faut le détourner de l'industrie chimique pour le braquer sur la surpopulation et l'agriculture.

Lovelock, comme de nombreux acteurs dans les années 1960 et 1970, et de nombreux acteurs ensuite liés au débat sur $\mathrm{HG}$, considère que le problème central vient de la surpopulation. On trouve dès 1975 des phrases qui rappellent celles de La revanche de Gaïa :

« Notre priorité en tant qu'espèce est de choisir parmi les nombreux moyens techniquement possibles pour limiter notre population ceux qui sont acceptables socialement en termes sociaux et moraux. ${ }^{87}$

Pour Lovelock, la surpopulation ne se manifeste pas par l'ensemble des activités métaboliques, incluant les activités des industries chimiques. Elle se manifeste toujours de la même manière : via l'agriculture. Le surplus d'humains se traduit par un surplus de bouches à nourrir, qui se traduit par une agriculture croissante, qui perturbe Gaïa.

La liste des maux attribués à l'agriculture - et parallèlement épargnés aux industries chimiques et pétrolières - est tout à fait sidérante. II n'est pas question pour nous d'adopter une posture asymétrique consistant à sous-estimer les impacts de l'agriculture sur le système Terre, devant ceux imputables aux industries chimiques, au motif que la première serait connotée de manière plus positive que la seconde.

Ce qu'il faut montrer en revanche, c'est à quel point les positions de Lovelock sont, elles, asymétriques. Ses dénonciations de l'agriculture ne reposent pas sur des démonstrations scientifiques abouties montrant effectivement les problèmes très réels posés par l'agriculture. Plutôt, l'agriculture constitue une cible prédéfinie à l'avance. Notre argument ne peut être démontré que d'une seule manière : en accumulant les cas. Faute de place, j'en livre ici un exposé ramassé 88 .

Dans un article paru dans Atmospheric Environment, l'agriculture est mise en cause par le mécanisme suivant (sans évaluation quantitative): l'augmentation de la poussière atmosphérique émise par l'agriculture à mesure que les tracteurs ont remplacé les chevaux et les bœufs et ont labouré plus profondément la terre. Et Lovelock conclut :

« Nous pourrions trouver finalement que les aspects directs de la combustion sont les moins nocifs de toutes les perturbations de l'homme sur l'écosystème planétaire, car le système pourrait avoir la capacité de s'adapter aux apports de gaz issus de la combustion. (...) Néanmoins, personne ne connaît les limites de l'adaptabilité de la Nature. II se pourrait que le point de rupture sera atteint en conséquence de l'activité perturbant les sols et élevant les poussières des agriculteurs tentant de satisfaire à la demande créée par l'explosion de la population, qui est indéniablement le résultat de l'activité humaine. ${ }^{89}$

En 1975, dans l'article sur Gaïa de New Scientist, les agriculteurs sont rendus responsables de la modification du climat et éventuellement de la couche d'ozone, via un autre mécanisme, là encore non évalué quantitativement :

« Les productions [d'oxydes d'azote] varient cependant, en conséquence de l'agriculture, et en particulier en conséquence de l'usage d'engrais azotés. Nous ne savons pas comment les oxydes d'azote pourraient modifier le climat, mais des preuves suggèrent qu'ils ont augmenté en

\footnotetext{
87 Lovelock et Epton, «The quest ». Op. cit.

88 Pour un développement, je renvoie à : Dutreuil, Gaïa. Op. cit., p. 674-680.

89 Lovelock, « Air pollution ». Op. cit., p. 410-411.
} 
concentration et ils sont connus pour pénétrer la stratosphère où leurs produits de décomposition pourraient affecter la couche d'ozone. ${ }^{90}$

En 1975 toujours, lors de la conférence sur le climat mentionnée plus haut, les agriculteurs constituent encore la cible centrale pour une nouvelle raison : I'agriculture pourrait perturber les cycles globaux de la matière si elle était étendue aux marais et aux plateformes continentales, et, ce faisant, également participer à la perturbation du climat et de la couche d'ozone :

"Bien plus sérieux que les dommages liés au 'hasard aveugle' de la pollution est l'activité intentionnelle et biocidaire de l'agriculture. Les habitants des villes, qui incluent la plupart des scientifiques, tendent à oublier que l'agriculteur moderne regarde toutes les créatures vivantes autres que ses cultures et son bétail comme des mauvaises herbes, des parasites et de la vermine à détruire. " $^{91}$

En 1977, un nouveau danger lié à l'agriculture est proposé par Lovelock et pourrait être responsable de la destruction de la couche d'ozone :

"L'océan pourrait être une source de chlorométhane pour l'atmosphère, mais il y a une autre source, la combustion lente des matériaux végétaux. Grossièrement, $10 \%$ du chlore contenu dans la végétation ainsi brûlée est converti en chlorométhane. Maintenant, il est possible que les feux de forêts et d'herbes des régions tropicales résultant de l'agriculture sur brûlis qui y est pratiquée soit l'autre source majeure de ce gaz dans l'atmosphère. " $^{92}$

Cet exemple est repris dans son livre sur Gaïa de 1979 pour dénoncer les conséquences d'une " agriculture primitive » (I'agriculture sur brûlis), au moins comparables aux effets de l'activité industrielle urbaine ${ }^{93}$.

Dans les années 1980, c'est encore un nouveau problème qui est identifié lorsqu'il s'intéresse au rôle des forêts tropicales dans Gaïa :

"Si l'évapotranspiration ou les mouvements de matériaux vers les océans via les rivières sont vitales pour l'homéostasie présente, alors le remplacement des forêts par l'agriculture ou des substituts de pâturages non seulement priverait de leurs régions les habitants rescapés qui y habitent mais pourrait également tout aussi bien menacer le reste du système. Nous ne savons pas encore si les systèmes de forêt tropicale sont vitaux pour l'écologie planétaire présente. Ils pourraient être comme les forêts tempérées qui semblent remplaçables sans préjudice sérieux. 1 $^{94}$

Les dangers de l'agriculture ne connaissant pas de limite, un nouvel effet pervers est identifié : en augmentant les intrants déversés in fine dans la mer, l'agriculture pourrait doper la productivité planctonique et ce faisant stimuler production de DMS qui pourrait être responsable des pluies acides ${ }^{95}$.

L'obsession de Lovelock vis-à-vis de l'agriculture en vient à prendre des accents théologiques :

90 Lovelock et Epton, "The quest ». Op cit., p. 306.

91 James Lovelock, "The interaction of the atmosphere and the biosphere. ", in William W. Kellogg et Margaret Mead, The atmosphere : endangered and endangering, Washington, D.C., U.S. Government Printing Office, 1977, p. 120.

92 James Lovelock, "Halogenated hydrocarbons in the atmosphere ", Ecotoxicology and environmental safety, vol. 1, n 3, 1977, p. 405.

${ }^{93}$ Lovelock, Gaia. Op. cit., p. 120.

94 James Lovelock, "Geophysiology: a new look at earth science. ", Bulletin Of The American Meteorological Society, vol. 67, $n^{\circ} 4,1986$.

95 Lovelock, The Ages. Op. cit., p. 152-154, 167 ; Lovelock, Gaia: medicine. Op. cit., p. 161 
« Je pense que de loin le plus grand dommage que nous faisons à la Terre, et par conséquent de loin la plus grande menace pour notre propre survie, vient de l'agriculture. (...) Quand nous pratiquons l'agriculture, à moins que nous le faisions très délicatement, nous nous échappons de notre obligation contractuelle avec Gaïa - et l'essentiel de l'agriculture, en particulier l'agro-business, est scandaleusement indélicate. D'une certaine manière, quand nous choisissons d'être des agriculteurs, nous cassons notre lien avec Gaïa et tombons du paradis. ॥" 96

La question n'est pas de savoir si Lovelock avait raison, dans le contexte scientifique de l'époque, de souligner les problèmes globaux engendrés par l'agriculture ni celle de savoir quel type d'agriculture est dénoncé ${ }^{97}$. Elle est de constater la diversité des maux attribués à l'agriculture et ce, le plus souvent, sur la base de mécanismes plus qu'hypothétiques, rarement accompagnés d'estimations quantitatives ou de citations scientifiques ${ }^{98}$.

Le lien que les prescriptions de Lovelock entretiennent avec Gaïa est ainsi ambivalent. D'un côté, les prescriptions sur l'agriculture sont sous-tendues par une analyse géophysiologique du globe (suivant le terme que Lovelock affectionne lorsqu'il se pose en médecin planétaire, quels que soient par ailleurs les mérites scientifiques de cette analyse) : e.g. faites ce que vous voulez des forêts tempérées, mais ne touchez pas aux forêts tropicales parce qu'elles sont essentielles pour le fonctionnement de Gaïa. Les prescriptions de Lovelock tranchent donc avec celles de ceux qui voudraient protéger toutes les parcelles sauvages de la Terre : elles sont sous-tendues par une vision fonctionnelle du globe, ainsi que Marshall l'avait déjà justement fait remarquer ${ }^{99}$.

Mais d'un autre côté, et c'est ce qui a échappé jusque-là aux commentateurs, aucun argument venant de l'hypothèse ou de la théorie Gaïa n'impose que la cause des problèmes globaux doit être recherchée systématiquement dans l'agriculture plutôt que dans l'effet de l'activité d'industries chimiques ${ }^{100}$.

L'interprétation des motivations qui ont sous-tendu l'asymétrie de Lovelock est délicate. Ces raisons tiennent sans doute pour une large part au choc émotionnel qu'a causé la révolution agricole dans la région qu'il habitait. Lovelock évoque ce choc dès la deuxième page de son autobiographie :

« Je veux vous expliquer dans ce livre pourquoi j'ai été m'enterrer dans le village de campagne de Bowerchalke. J'y ai travaillé de manière heureuse jusqu'en 1977, quand malheureusement la

\footnotetext{
96 Lovelock, Gaia : medicine. Op. cit. p. 156.

97 Lovelock dénonce bien sûr certains types d'agriculture: l'agriculture industrielle, l'agriculture " primitive» sur brûlis. Mais il affirme également que l'agriculture industrielle est nécessaire, parce qu'elle est plus productive et donc plus économe en surface de Terre.

98 Le cas le plus frappant est celui des pluies acides. Lovelock reconnaît à demi-mot que le débat scientifique s'accorde pour dire que la cause principale doit être recherchée dans le soufre issu des combustibles fossiles. Mais il ne peut s'empêcher de réfléchir au rôle de l'agriculture. Pour une réfutation quantitative de la spéculation de Lovelock, voir: Caroline Leck et Henning Rodhe, " Emissions of marine biogenic sulfur to the atmosphere of northern europe. ", Journal of atmospheric chemistry, vol. 12, n 1, 1991, p.63-86.

99 Alan Marshall, The unity of nature: wholwhole and disintegration in ecology and science, London, Imperial College Press, 2002, Chapitre 3.

100 Lovelock ne peut aucunement ignorer que d'autres causes que l'agriculture sont au cœur des controverses. Dans les années 1960, il n'était pas admis que le climat allait se réchauffer en raison de l'émission de gaz à effet de serre issus des combustibles fossiles. Certains arguments suggéraient de possibles glaciations, résultant d'une augmentation de la réflectivité de l'atmosphère augmentée par les aérosols issus de la combustion d'énergies fossiles. Lovelock n'ignore pas ces débats : en juin 1966 il rédige une note confidentielle pour Shell intitulée "Combustion of fossil fuel : large scale atmospheric effect » détaillant ces deux mécanismes et concluant : "Ce qui semble être important, ce ne sont pas tellement les explications et si elles sont correctes dans les détails mais plutôt le fait presque certain que le climat se dégrade et la probabilité que la combustion de combustibles soit responsable. " [Archives de Lovelock].
} 
révolution de l'agro-business a purgé socialement le village. Mon échappatoire a été dans le Devon ouest (...). »101

Ironie de l'histoire, il sait de quoi il parle lorsqu'il déplore la suppression du bocage en Angleterre, puisqu'il en a indirectement été un des artisans. II a en effet personnellement participé aux programmes de recherches du Grassland Research Institute dans les années 1940 suggérant des modifications aux agriculteurs, comme de supprimer les haies, pour améliorer les rendements, et ces recherches allaient ensuite informer le Ministère de l'Agriculture et se déployer à grande échelle.

\section{Conclusions historiques et perspectives philosophiques}

Gaïa occupe donc une place tout à fait centrale dans l'histoire de l'émergence des problèmes et des savoirs de l'environnement global. Pour en prendre la mesure, il faut premièrement sortir d'une lecture répandue mais erronée sur deux points essentiels : HG ne doit pas être lue comme une controverse théorique avortée et interne à la biologie de l'évolution mais comme un nouveau programme de recherche pour les sciences de la Terre, ayant contribué à la constitution des sciences du système Terre; HG n'a pas intéressé quelques quelques marginaux néo-païens mais bien des acteurs au centre des mouvements environnementalistes et de l'écologie politique.

Mais il faut également sortir de l'historiographie véhiculée par Lovelock suivant laquelle HG serait le produit de réflexions spéculatives et abstraites sur la vie d'un scientifique indépendant et coupé du monde, reclus dans son garage du Devon. Si Gaïa a pu prendre une telle importance, c'est précisément parce que Lovelock était au centre d'un réseau particulièrement dense couvrant des institutions décisives pour l'orientation des recherches en sciences de l'environnement et de la Terre - la NASA, la NOAA, le NCAR, ou encore, pour celles que nous n'avons pas couvertes ici, le MetOffice et le Natural Environment Research Council britanniques - mais aussi les industries chimiques et pétrolières - Shell, HP, DuPont, etc. - au centre des dégradations environnementales globales - DDT, CFC, changement climatique, smog, pollutions au plomb, pluies acides, etc. - et les acteurs des mouvements environnementalistes, de l'écologie politique et de la contreculture environnementale, jusqu'à des personnalités politiques.

Le travail de Lovelock comme ingénieur et chimiste, et notamment comme inventeur de I'ECD et pionnier dans certains domaines de chimie de l'environnement, l'a placé au centre des sciences de l'environnement et des industries chimiques. La crédibilité scientifique acquise par Lovelock lui a permis de donner une audience large à Gaïa en climatologie et en chimie de l'atmosphère. Parallèlement l'élaboration de Gaïa comme nouvelle conception de la nature lui a ouvert les portes des mouvements environnementalistes. Margulis, reconnue pour ses travaux fameux sur l'endosymbiose, a contribué de manière importante à consolider et élargir ce réseau auprès de l'écologie, de la microbiologie et de l'histoire de la Terre.

Pour comprendre l'intensité de ces liens et le caractère central de Gaïa, il ne suffit pas de s'intéresser à la place des acteurs dans ces réseaux. Il est aussi nécessaire, sur le plan conceptuel et théorique, de relire Gaïa non pas comme une hypothèse scientifique locale, interne à un champ, mais bien comme une nouvelle philosophie de la nature visant à reconfigurer certaines catégories importantes comme celles de nature, de vie, d'environnement et de pollution. C'est bien la reconnaissance d'une nouvelle entité, Gaïa, qui a joué à la fois un rôle fondateur pour les sciences du système Terre et a été à la base d'une nouvelle conception de la nature pour l'écologie politique. Enfin, cet article propose de

101 Lovelock, Homage. Op. cit., p. 2. Dans le second livre de Lovelock, c'est un épilogue de 10 pages qui est consacré à la campagne anglaise et aux transformations délétères apportées par la révolution agricole: Lovelock, The Ages. Op. cit. Et jusque dans ces derniers livres, il dit toute son amertume à propos de la mécanisation de l'agriculture et la destruction de la campagne sauvage anglaise et du bocage : Lovelock, The Vanishing, Op. cit., p. 220. 
comprendre la genèse historique de cette philosophie de la nature d'abord et avant tout comme celle d'un cadre conceptuel pour penser les phénomènes de pollution globale.

Du point de vue de l'histoire et la sociologie des sciences, cet article montre également que la figure du " scientifique entrepreneur » de Shapin n'est aucunement restreinte aux start-ups de biotech de la Silicon Valley : dans les sciences de la Terre et de l'environnement, au cœur des années 1970, des scientifiques comme Lovelock ont également un pied dans la production de savoirs académiques et un autre dans des entreprises privées directement concernées par ces savoirs académiques.

Cette analyse historique peut alimenter et nourrir les questions de philosophie de la nature qui se sont articulées récemment autour de Gaïa ou de l'anthropocène. Ce n'était pas l'objet de cet article, nous nous contentons donc ici de perspectives.

HG a opéré des déplacements importants sur la manière dont est classiquement pensé le milieu ou dont sont habituellement pensées les relations vie/environnement en écologie et en biologie de l'évolution : au sein d'HG, le milieu n'est pas considéré comme un ensemble de ressources locales que les organismes consomment pour croître, mais comme un ensemble de variables globales, affectées dans un sens ou dans l'autre par les activités des vivants, et notamment leurs rejets de déchets métaboliques ${ }^{102}$.

Latour a souligné que Gaïa contribuait à une relocalisation de la nature, remettant en question la philosophie de la nature galiléenne : fini le cosmos infini, retour sur Terre dans un monde sublunaire animé ${ }^{103}$. Les éléments historiques présentés ici renforcent sans doute le parallèle avec Galilée. La philosophie galiléenne de la nature doit beaucoup au réseau de puissants dont était entouré Galilée ${ }^{104}$; de même le contenu et le succès de Gaïa ne sont pas étrangers à l'étendue du réseau de Lovelock $^{105}$.

Ces éléments historiques apportent en outre des éclairages sur les débats contemporains autour de l'anthropocène en suggérant les nombreux points de connexion institutionnels, historiques et philosophiques entre Gaïa et l'anthropocène. Ce terme est apparu en 2000, au sein de l'IGBP, I'institution qui avait émergé dans les années 1980 au moment de la constitution des sciences du système Terre ${ }^{106}$. Les hérauts respectifs de Gaïa et de l'anthropocène, Crutzen et Lovelock, se connaissent depuis les années 1970, à une époque où ils travaillaient tous les deux sur les CFCs et l'ozone. Enfin, l'ontologie de l'anthropocène, contrairement à ce que son nom indique, hérite moins de celle de la stratigraphie et de l'histoire de la Terre, que de la conception systémique de la Terre mise en avant par Gaïa ${ }^{107}$.

Pour finir, le sol constitue un point nodal intéressant dans l'histoire des conceptions holistes et systémiques de la Terre que certains font remonter à la Théorie de la Terre du géologue écossais James Hutton ${ }^{108}$ ou à La biosphère du savant russe Vladimir Vernadsky ${ }^{109}$. Ainsi, on

102 Dutreuil, Gaïa. Op. cit.; Sébastien Dutreuil et Arnaud Pocheville, « Les organismes et leur environnement: la construction de niche, I'hypothèse gaïa et la sélection naturelle ", Le Bulletin d'histoire et d'épistémologie des sciences de la vie, vol. 22, n 1, 2015, p. 27-56.

103 « II est difficile de ne pas être frappé par la symétrie renversée entre les gestes de Galilée et de Lovelock levant de modestes instruments vers le ciel pour y faire des découvertes radicalement opposées. (...) Tandis que Galilée, levant les yeux de l'horizon vers le ciel, renforçait la similitude entre la Terre et tous les autres corps en chute libre, Lovelock, baissant les yeux à partir de Mars dans notre direction, diminue en fait la similitude entre toutes les planètes et cette Terre si particulière qui est la nôtre ", Latour, Face. Op. cit., p. 102, 105.

104 Mario Biagioli, Galileo, courtier: The practice of science in the culture of absolutism, Chicago, University of Chicago Press, 1993.

105 Je dois ce prolongement à une remarque de Bruno Latour.

106 Clive Hamilton et Jacques Grinevald "Was the anthropocene anticipated? " The anthropocene review, vol. 2, n 1, 2015, p. 59-72 ; Dutreuil, Gaïa. Op. cit.

107 Sébastien Dutreuil « L'anthropocène est-il un concept d'histoire de la terre ? Le nom qui ne dit pas son épistémologie ", in Rémi Beau et Catherine Larrère, Penser l'anthropocène? Paris, Presses de Sciences Po, à paraître.

108 James Hutton. Theory of the Earth with proofs and illustrations. Four volumes. Londres, Cadell, Junior, 
sait à quel point la question de la régénération du sol était centrale dans l'élaboration de la théorie de la Terre de Hutton. De même, Vernadsky a d'abord été formé par un pédologue russe, Dokuchaev, avant de devenir un des fondateurs de la géochimie et un théoricien de la biosphère au début du $20^{\mathrm{e}}$ siècle. Mais, ainsi que nous l'avons vu dans la dernière section, alors qu'on pensait que Gaïa était de part en part une philosophie de l'atmosphère, nous voilà, avec Lovelock, revenir par des chemins détournés à la question de l'agriculture et de l'usage des sols.

Davies, 1795

109 Vladimir I. Vernadsky, La biosphère, Paris, Seuil, 2002 [1929]. 